blood volume either from extravasation into tissue spaces locally or generally, for example, in shock produced by anthrax, or from a peripheral pooling of the blood and a decrease in venous return as, for example, in shock produced by staphylococcal and meningococcal toxins. The possible biochemical mechanisms whereby different bacterial products affect blood vessels is a relatively untouched field for research.

W. W. Spink (United States), in his paper on "Brucellosis as a model for metabolic studies on bacterial shock and inflammation," directed attention to the increasing interest shown in recent years in the peripheral vascular collapse caused by endotoxins of Gram-negative bacteria. There is vasoconstriction of the small vessels, which causes ischæmia of the tissues, and changes in cellular function result. This is followed by vasodilation and stagnation of blood, increased vascular permeability, and œdema and hæmorrhage. There is reduced cardiac output because of peripheral pooling of blood. Renal failure occurs because of the compensatory vasoconstriction, which results in ischæmic tubular necrosis.

The metabolic response of the host to bacterial invasion includes alterations in hormonal activity. The adrenal cortex is stimulated, as reflected by increased concentrations of adrenocorticoids, especially cortisone. The thyroid stimulates the activity of the adrenal cortex. Some doubt was expressed as to the wisdom of giving the large amounts of exogen. ous corticosteroids advised by Dr. Spink during the initial critical period of endotoxin shock. It was of interest that he had found that triiodothyronine accelerated the lethal action of endotoxin.

"The bacterial factor in traumatic shock" was discussed by J. Fine, E. D. Frank, H. A. Ravin, S. H. Rutenburg and F. B. Schweinburg (United States), who found evidence of a toxin appearing in the blood of animals in hæmorrhagic shock, such toxin having many of the hallmarks of a bacterial endotoxin. They consider that it is the cause of the irreversibility to transfusion in their standardized preparations. A toxin with similar properties to the one in the blood in hæmorrhagic shock is apparently present in the blood of the animal in tourniquet shock and in non-bacteræmic septic shock. The source of this toxin appears to be such Gram-negative bacteria as may be present in the animal's tissues, and the endotoxins normally present in the gastro-intestinal tract, which appear to enter the circulation at a steady rate, if only in trace quantities. It is thought that when shock, however produced, becomes unresponsive to therapy, the refractory state of the peripheral vessels is due to unneutralized endotoxins through impairment of the reticulo-endothelial system. The protective effect of reserpine and of dibenamine given prophylactically lend strength to the view that the endotoxins act adversely on these vessels by intensifying the local action of adrenaline or noradrenaline.

S. M. Rosenthal and H. Tabor (United States), in their paper on "Some aspects of therapy of injury", described the survival effects of administering isotonic sodium salts to mice subjected to what would otherwise be one lethal dose of burn trauma, tourniquet trauma or hæmorrhage. In their estimation, sodium and fluid depletion are the major factors in the cause of shock. Saline or plasma in amounts of 5 per cent of body-weight ( $50 \mathrm{ml} . / \mathrm{kgm}$.) is inadequate. These authors have been able to demonstrate a specific effect from the sodium ion in shock. Potassium salts behave in an opposite manner to sodium and are quite toxic. In shocked mice there is an eightfold increase over the toxicity in normal. Glucose and colloid solutions containing no sodium were relatively ineffective in the therapy of shock. The concept that plasma proteins exert their osmotic effects by being held rigidly in the vascular bed must be modified by the demonstration of Wasserman and Mayerson in 1952 that a complete extravascular circulation of the plasma proteins occurs every 20 hours.

In hæmorrhage and hæmorrhagic shock where there is a large loss of red cells, the striking efficacy of administering red cells has been clearly demonstrated, but there is little evidence to substantiate its value in shock from burn or other local trauma. In experimental burns and tourniquet shock whole blood is no more effective than plasma or saline solutions.

In studies on burned children in Peru a small but statistically significant benefit from plasma above that from saline was observed. The important problem of late deaths following severe burns is also being studied in Peru by Drs. Rosenthal and Millican.

This, the first main symposium covering this field of investigation to be held in Europe since the Second World War, was of great value to the participants. The papers contributed and the discussion which followed will be published in due course by Blackwell Scientific Publications, Ltd.

D. P. Cuthrertson

\title{
FREE RADICAL STABILIZATION
}

$\mathbf{F}$ REE radical chemistry began with the discovery of the stable aromatic free radicals by Gomberg in 1900. The second stage of the subject developed more gradually as the occurrence and importance of unstable free radicals became increasingly apparent and as kinetic and spectroscopic methods for their study in the gas phase and in solution were perfected. During the past few years a third chapter in free radical chemistry has been opened with the realization that even the most reactive of radicals can readily be stabilized for indefinite periods by trapping them in a rigid matrix at low temperatures.
An informal discussion of the Faraday Society on this subject was held in the Chemistry Department of the University of Sheffield on September 4 and 5. The meeting was attended by about one hundred and eighty scientists, half of whom were from Britain, fifty from the United States and Canada, and forty from other countries. Twenty-one papers, covering nearly all aspects of the subject, were presented and discussed.

The three principal methods of preparation of stabilized radicals are: (1) by irradiation of a rigid solid or solution at low temperatures; (2) by elec- 
trical or thermal dissociation of a gas and subsequent trapping on a cold surface ; (3) by chemical reaction in a rigid or viscous medium, for example, polymerization.

The trapped radicals are then investigated by physical methods, the most useful being absorption spectroscopy in the infra-red, visible or ultra-violet regions, thermal analysis and electron paramagnetic resonance spectroscopy. The latter method is particularly appropriate, and it is fortunate that it was developed at about the same time as methods for the stabilization of radicals.

Practically every type of radical has now been stabilized by one of these methods, from atoms and diatomic radicals to complex odd-electron molecules in polymeric and biological systems. Although the subject is at an early stage of development, the identification of many of these radicals is now quite certain. The simplest of all-the hydrogen atom-is readily prepared and identified by means of its characteristic electron spin resonance doublet, and detailed studies of its formation by gamma irradia. tion of acid solutions at $77^{\circ} \mathrm{K}$. were reported by $R$. Livingston, H. Zeldes, A. Weinberger and R. Holmberg (Oak Ridge National Laboratory). 'The isolation of other atomic species is less well established. Unstable products are undoubtedly present in the condensed products of an electrical discharge through nitrogen or after $\gamma$-irradiation or electron bombardment of the solid at $4^{\circ} \mathrm{K}$. (E. M. Hörl, National Bureau of Standards, Washington), but the evidence at present suggests that, although nitrogen atoms have been detected, $\mathrm{N}_{3}$ may be the main constituent under some conditions (L. A. Wall, D. W. Brown and R. E. Florin, National Bureau of Standards; also K. Jen, Johns Hopkins University, Maryland).

The first work on the stabilization of simple inorganic free radicals was that of F. O. Rice (Catholic University, Washington), who found a transient blue substance, attributed to the imine radical, in the condensed products of dissociation of $\mathrm{NH}_{3}$. Rice illustrated this early experiment, as well as later work, by means of excellent colour films which he recommended as being more reliable than the actual experiment for demonstrations of this kind. G. Wilse Robinson and M. McCarty (Johns Hopkins University, Baltimore) reported some very interesting and successful work on the stabilization of diatomic and triatomic radicals at $4^{\circ} \mathrm{K}$. The radicals $\mathrm{NH}, \mathrm{OH}$, $\mathrm{CH}, \mathrm{C}_{2}, \mathrm{NH}_{2}, \mathrm{HNO}, \mathrm{HCO}$ and CNO prepared in the electrical discharge, have been positively identified in a rigid argon matrix by means of their electronic absorption spectra. The spectra are surprisingly sharp and reveal that radicals such as $\mathrm{NH}_{2}, \mathrm{NH}$ and $\mathrm{OH}$ experience nearly free end-over-end rotation in the matrix.

Most successful work on the aliphatic radicals has utilized electron spin resonance methods since their electronic spectra lie in the far ultra-violet, and infra-red methods present many problems. For example, H. W. Brown (National Bureau of Standards) was not able to obtain the infra-red spectrum of methyl by photolysis of methyl iodide, but K. Jen successfully used this method to record its electron spin resonance spectrum. M. S. Matheson (Argonne, National Laboratory) summarized the radicals which have so far been identified in the irradiation products of paraffins, alcohols, ethers, aldehydes, ketones, fatty acids and other aliphatic compounds. The electron spin resonance spectra of the simple alkyl radicals are now well established. The ethyl radical is formed by the $\gamma$-irradiation not only of ethane but also of ethylene, and R. Klein and M. D. Scheer (National Bureau of Standards) described studies of the reactions of hydrogen atoms with solid olefins at $77^{\circ} \mathrm{K}$. which show that addition occurs readily under these conditions. The $\gamma$-irradiation of alcohols and ethers is very specific in removing almost exclusively the hydrogen atom from the carbon $\alpha$ to the oxygen. D. J. E. Ingram and M. C. R. Symons (University of Southampton) described how the same radicals are also formed by photolysis of a solution of hydrogen peroxide in the rigid alcohol; the $\mathrm{OH}$ radical first formed readily abstracts hydrogen from the alcohol at $77^{\circ} \mathrm{K}$.

For the aromatic radicals electronic absorption spectroscopy has proved to be most useful. H. T. J. Chilton G. Porter (University of Sheffield) and E. E. Strachan (British Rayon Research Association) described the preparation and stabilization of a variety of such radicals both by photolysis and by gamma radiolysis of rigid solutions at $77^{\circ} \mathrm{K}$. In the case of the ionizing radiations the dissociation of the aromatic molecules is brought about almost exclusively by transfer of energy absorbed by the solvent.

The irradiation of polymers leads to larger radicals, and these are often still present at normal temperatures. For example, V. V. Voevodsky (Academy of Sciences, U.S.S.R.) described electron spin resonance studies of radicals trapped in irradiated poly. tetrafluoroethylene at $77^{\circ} \mathrm{K}$., some of which remain when the specimen is warmed to $20^{\circ} \mathrm{C}$. Radicals are also trapped in polymers as a result of the polymerization reaction itself and are responsible for unusual and characteristic kinetic behaviour. C. E. H. Bawn, T. P. Hobin and W. J. MeGarry (University of Liverpool) described studies of trapped radicals in acrylonitrile, vinyl chloride, styrene and vinylidene chloride.

The general occurrence of stabilized radicals in polymeric and biological systems was reviewed by C. H. Bamford and A. D. Jenkins (Courtaulds, Ltd., Maidenhead). Electron spin resonance measurements have shown that metabolically active plant and animal tissues such as leaves, roots, seeds, blood, muscle and liver have a remarkably high radical content, and W. Gordy (Duke University) described recent investigations on such systems. Their function and the means by which they are stabilized and, indeed, their chemical nature are little understood as yet. J. L. Vivo-Acrivos and M. S. Blois (Stanford University, California) reported on electron spin resonance studies of stable radicals in natural and synthetic melanins. D. K. Ghosh and D. H. Whiffen (University of Birmingham) described their detailed work on the electron spin resonance spectra of radicals trapped in a single crystal of glycine after gamma irradiation. It can be shown that the radical is $\mathrm{NH}_{3}$.CH.COO - . The spectrum is anisotropic and shows far more detail than that from a polycrystalline material. The arrangement of the trapped radicals has the same symmetry as that of the molecules in the host lattice.

Although the major effort in the field of radical stabilization is at present directed towards the identification of the trapped species, it is clear that, once the spectra have been assigned, the method will provide a powerful means of investigation in many fields, and particularly of the primary processes of photochemistry, radiation chemistry and the electrical discharge. Masterly reviews of these three 
aspects of the subject were given by $\mathrm{H}$. Linschitz (Brandeis University), M. S. Matheson and H. P. Broida. There was considerable discussion of the characteristic behaviour of excited states in rigid media, and Broida stressed the need for more information about the matrix structure and about the fundamental limitations to trapped radical concentrations.

There has been a great deal of interest in the possible use of stabilized radicals as high-energy rocket propellants. The important quantity to be considered is the heat of reaction per gram, and there seems little hope of increasing this greatly above $3 \mathrm{kcal} . \mathrm{gm}$. with conventional molecular fuels. If pure atomic hydrogen could be utilized, the heat of reaction would be $5 \mathrm{l} \cdot 2 \mathrm{kcal} . / \mathrm{gm}$., but the present indications are that concentrations which can be trapped are limited to 1 per cent or less. Other, heavier radicals are all much less suitable, and G. C. Szego (General Electric Co., Cincinnati) concluded that, at present, this application of stabilized radicals is not promising. Some enthusiasm was shown about the possibility of utilizing metastable excited states, such as the triplet state of helium, which has an excess energy of $113 \mathrm{kcal} . / \mathrm{gm}$.

Special experimental techniques are mainly concerned with the adaptation of existing methods to low temperatures. L. J. Shoen, L. E. Kuentzel and H. P. Broida (National Bureau of Standards) described glass Dewar flasks for optical studies at low temperatures, P. Le Goff and L. P. Blanchard (University of Nancy) described a mass spectrometer with a lowtemperature ionization chamber, and H. S. Peiser (National Bureau of Standards) gave an account of his very elegant X-ray crystallographic studies on low-temperature solids.

The enthusiasm of the discussions was typical of a field where new phenomena are found in nearly every experiment which is performed. The international character of the meeting was a very pleasant feature, and this was assisted by financial support from the United States Department of the Army through its European research office.

George Porter

\title{
NEW POTENT REACTIVATORS OF ACETOCHOLINESTERASE INHIBITED BY TETRAETHYL PYROPHOSPHATE
}

\author{
By Dr. F. HOBBIGER \\ Department of Pharmacology, \\ AND \\ Dr. D. G. O'SULLIVAN and DR. P. W. SADLER \\ Courtauld Institute of Biochemistry, \\ Middlesex Hospital Medical School, London, W.I
}

\begin{abstract}
CERTAIN organophosphates, for example, tetra( ethyl pyrophosphate (TEPP), ditsopropyl phosphonofluoridate (DFP), diethyl $p$-nitrophenylphosphate $(E 600)$, and a number of widely used insecticides and their metabolites, are potent inhibitors of acetocholinesterase. Organophosphate poisoning (fatal when sufficient acetylcholine has accumulated in the body) has been reported from many countries, especially from Japan ${ }^{1}$ and the United States ${ }^{2}$, and an efficient treatment has become essential. Acetocholinesterase is reputed to possess positively and negatively charged regions, commonly called anionic and esteratic sites, which participate in the hydrolysis of acetylcholine. In the inhibition process, a dialkylphosphoryl group, or some related group, becomes attached to the esteratic site and in this way deprives the enzyme of its hydrolytic func. tion $^{3}$. Spontaneous reactivation of diethyl phosphoryl-acetocholinesterase occurs very slowly, but the formation of diisopropyl phosphoryl-acetocholinesterase is, for all practical purposes, irreversible 4 .

Certain nucleophilic reagents restore the activity of freshly phosphorylated acetocholinesterase by displacing the phosphoryl group from the enzyme ${ }^{3}$. Hitherto, pyridine-2-aldoxime methiodide has proved, in general, to be the most potent reactivator, and attachment of its quaternary nitrogen to the anionic site of the phosphorylated enzyme has been considered to contribute to its potency ${ }^{5}$.

During the past two years we have synthesized a large number of oximes and studied the affinity
\end{abstract}

of these compounds for acetocholinesterase, their interaction with tetraethyl pyrophosphate, and their effectiveness in restoring the activity of acetocholinesterase inhibited by tetraethyl pyrophosphate. The compounds can be classified roughly as aliphatic oximes, and compounds related to pyridine, indole, indolinene, quinoline and isoquinoline. The most powerful reactivators that have so far emerged are structurally related to pyridine, the effectiveness of some of these being shown in Tables 1 and 2. Monoximes and dioximes of bis-pyridinium ions (compounds 12-18; Table 2) are all considerably more potent reactivators than pyridine-2-aldoxime methiodide $(P-2-A M)$ although they are derivatives of the less active pyridine-4-aldoxime methiodide $(P-4-A M)$. A considerable gain in activity is also obtained by terminal halogen substitution in the alkyl side-chain (compare compounds 3 and 8 ). Table 2 shows that the reactivating power of the oximes cannot be predicted from their hydrolytic activity and their affinity for the anionic site of acetocholinesterase. The order of activities found :

$$
P \cdot 2 \cdot A M>P \cdot 4 \cdot A M \gg P \cdot 3 \cdot A M
$$

is in conformity with unpublished results obtained with other compounds, which suggests that a conjugative connexion between the quaternary nitrogen and an oxime or hydroxamic acid group is an essential feature for high activity.

All the compounds in Table 2 have been subjected to a preliminary screening for antidotal properties. 